\title{
Telescope Array Combined Fit to Cosmic Ray Spectrum and Composition
}

\section{Douglas R. Bergman ${ }^{a, *}$ on behalf of the Telescope Array Collaboration}

(a complete list of authors can be found at the end of the proceedings)

${ }^{a}$ Dept. of Physics \& Astronomy and High Energy Astrophysics Inst.,

University of Utah, Utah, USA

E-mail: bergman@physics.utah.edu

We present the result of fits of an astrophysical model of UHECR sources to the energy spectrum measured using the Telescope Array (TA) surface detectors and the composition data measured by TA fluorescence detectors in stereo mode. The astrophysical model consists of identical sources, uniformly distributed with a density which evolves with age, where groups of nuclei with varying fractions are accelerated producing a power law source with a rigidity-dependent cutoff. The model includes the propagation effects of photo-pion production, photo-nuclear disintegration and general energy loss from universal expansion. Comparison of model data with detector data in both the energy and the $X_{\max }$ distributions is done via a forward-folding description of detector effects. A prediction of the cosmogenic neutrino flux at Earth resulting from the cosmic rays produced in the model with these fit values is also presented. The predicted flux is considerably higher than other similar fits to Auger data have previously predicted.

$37^{\text {th }}$ International Cosmic Ray Conference (ICRC 2021)

July 12th - 23rd, 2021

Online - Berlin, Germany

\footnotetext{
${ }^{*}$ Presenter
} 


\section{Introduction and Model}

The origin of Ultra High Energy Cosmic Rays (UHECRs, $E>10^{18} \mathrm{eV}$ ) remains undetermined despite a sample of tens of thousands of events collected by very large observatories around the world. UHECRs are believed to be of extragalactic origin, because of the observed anisotropy of arrival directions and the diffusion scale for magnetic field within the galaxy.

Employing a relatively simple model of UHECR sources we use Telescope Array (TA) measurements of the energy spectrum and the observed distributions of $X_{\max }$, the depth in the atmosphere where extensive air showers reach their maximum size, to constrain possible UHECR source parameters.

The model we are employing is the same as that used by the Pierre Auger Observatory in a similar fit [1]. The source model assumes that UHECR sources are identical and uniformly distributed with a density which evolves as $(1+z)^{3}$ for $z<1.5$ and is constant for $z>1.5$ [8]. Each source has a mix of five nuclei $(\mathrm{H}, \mathrm{He}, \mathrm{N}, \mathrm{Si}$, and $\mathrm{Fe})$ that are accelerated to give spectra with identical power law slopes $\left(E^{-\gamma}\right)$ and a rigidity-dependent exponential cutoff, $R_{\max }$. The source parameters are therefore $\gamma, R_{\max }$, and five fractions (which sum to unity). Cosmic rays generated at $z>1$ cannot effect TA observations at the highest energies; however, cosmic rays from these distant sources contribute significantly to the predicted neutrino flux.

The model is constrained by the TA surface detector (SD) spectrum measurement (as presented at the 2017 ICRC [2]) and the TA Stereo Composition measurement (also from the 2017 ICRC [3]). We choose the TA SD spectrum because it has the largest set of statistics for a UHECR energy spectrum measured in the Northern Hemisphere. It is also different from the spectrum measured in the Southern Hemisphere with a higher energy for the final sharp break in the spectrum $(60 \mathrm{EeV} c f$. $40 \mathrm{EeV}$ ). We choose the TA Stereo composition measurement because of its indifference to high energy particle interaction models.

The effects of propagation of nuclear species through the universe is done by the CRPropa model [4]. The CRPropa calculation was done using both the cosmic microwave background (CMB) and the Gilmore model [5] of the infrared background (IRB) to calculate photo-pion production, photo-nuclear disintegration and electron-positron pair-production. The cosmological parameters used were those determined by Planck in 2015 [6]. A 1-D propagation calculation was performed for a series of small ranges in $z$, "shells", with 462 shells logarithmically spaced between $z=10^{-4}$ and $z=4$. 20 thousand particles per 0.1 decade of energy of each source species were injected with an $E^{-1}$ spectrum between the energies of $0.0316 \mathrm{EeV}$ and (rigidity dependent) $3160 \mathrm{EV}$. Each "observed" nuclei was recorded and binned by atomic number. Thus for each input species, each possible output species and each shell, we create a $650 \times 650$ bin $E_{\text {out }} / E_{\text {in }}$ histogram. ( $E_{\text {in }}$ is the injected energy, $E_{\text {out }}$ is the energy at observation) These can be combined into big arrays, e.g., for Fe $(A=56)$ we have a $56 \times 462 \times 650 \times 650$ array. As this gets to be an unruly size of data, we sum over the $462 z$-shell bins weighted by the light-travel time across the shell (i.e. the thickness of the shell) and the density of sources in the shell (given by the $z$-dependent density assumption given above). Using the $E_{\text {out }} / E_{\text {in }}$ histograms then we can put in any input (source) spectrum and get the expected "observed" spectrum of a particular nuclear type. The input spectrum is created according to the model given above.

For comparison with the spectrum we use this output directly. For comparison with the stereo 


\begin{tabular}{|l|c|c|c|c|c|c|}
\hline \hline Energy Range & $18.4-18.6$ & $18.6-18.8$ & $18.8-19.0$ & $19.0-19.2$ & $19.2-19.6$ & $19.6-20.0$ \\
\hline proton & 21.8 & 19.0 & 17.6 & 16.3 & 16.4 & 16.4 \\
Iron & 17.8 & 16.8 & 14.8 & 13.2 & 13.0 & 12.8 \\
\hline \hline
\end{tabular}

Table 1: Table of $X_{\max }$ resolutions in $\mathrm{g} / \mathrm{cm}^{2}$. Energy ranges are given in $\log _{10}(E / \mathrm{EeV})$

composition measurement we use the Gumbel [7] distribution to create $X_{\max }$ distributions for each nuclear type and energy. The parameters for the Gumbel distribution for given high-energy interaction models has been tabulated in [7]. We must then also apply a stereo $X_{\max }$ acceptance weighting to these distributions and smear according to the stereo $X_{\max }$ resolution. The $X_{\max }$ acceptance was calculated in the energy bins used and then fit to a model of a flat-top plateau, a breakpoint in $X_{\max }$ and an exponential fall-off in acceptance above the break point. It was found that a single value of the breakpoint and the exponential slope could be used to fit the acceptance in all energy bins. The $X_{\max }$ acceptance is shown in Figure 1. The stereo $X_{\max }$ resolution was calculated by comparing the generated and the reconstructed $X_{\max }$ values for simulated proton and iron primaries using in each energy band. This determination of the $X_{\max }$ resolution is the same as that determined from the stereo composition analysis [3]. The values for the $X_{\max }$ resolution used in this analysis are stated in Table 1. The $X_{\max }$ distribution resulting from the output of the Gumbel model and with the acceptance model applied, was then smeared using a single gaussian in $X_{\max }$ with a width given by logarithmic interpolation between the tabulated proton and iron resolutions based on the known primary nuclear mass. The sum of these $X_{\max }$ distributions for all the observed primary cosmic ray species was then compared to the observed $X_{\max }$ distribution.

For both the spectrum and composition analyses we use a binned log-likelihood comparison of events observed compared to events in the model. For the spectrum comparison the log-likelihood is based on the Poisson distributions, while for the $X_{\max }$ comparison, the log-likelihood is based on a multinomial distribution. Both log-likelihood comparisons are scaled to produce a $\chi^{2}$-like distribution in the limit of very large numbers of events. We refer to these as deviances, and sum the deviance from the spectrum and the deviance from the compositions analysis to determine an overall figure-of-merit for the model with given parameters.

\section{Combined Fit Results}

Our fits presented here use the QGSJetII-04 high-energy interaction model for the Gumbel /Xmax/ distributions and the Gilmore-12 IRB model. We scan the $\gamma-\log _{10}\left(R_{\max } / \mathrm{EV}\right)$ plane, and for each pair allow the five source fractions to vary under the requirement that the fractions sum to unity. We first performed coarse scans, $\gamma$ was scanned in 41 steps from $-1.5-2.5$ while $\log _{10} R$ was scanned in 41 steps from $-0.5-3.5$. To allow for systematic uncertainties in energy and $X_{\max }$ reconstruction, we shifted the measured energy and $X_{\max }$ of the data by various amounts, adding a $\chi^{2}$-penalty to the deviance to account for the shifts. The systematic uncertainty in the energy measurement was taken to be $22 \%$ while the systematic uncertainty in the $X_{\max }$ measurement was taken as $15 \mathrm{~g} / \mathrm{cm}^{2}$. The best fits were found with $\Delta \log _{10} E=-0.12(1.4 \sigma)$ and $\Delta X_{\max }=+10 \mathrm{~g} / \mathrm{cm}^{2}$ $(0.67 \sigma)$. We then performed a fine scan with these shift values, 401 steps for $\gamma$ from $-1.5-2.5$, 

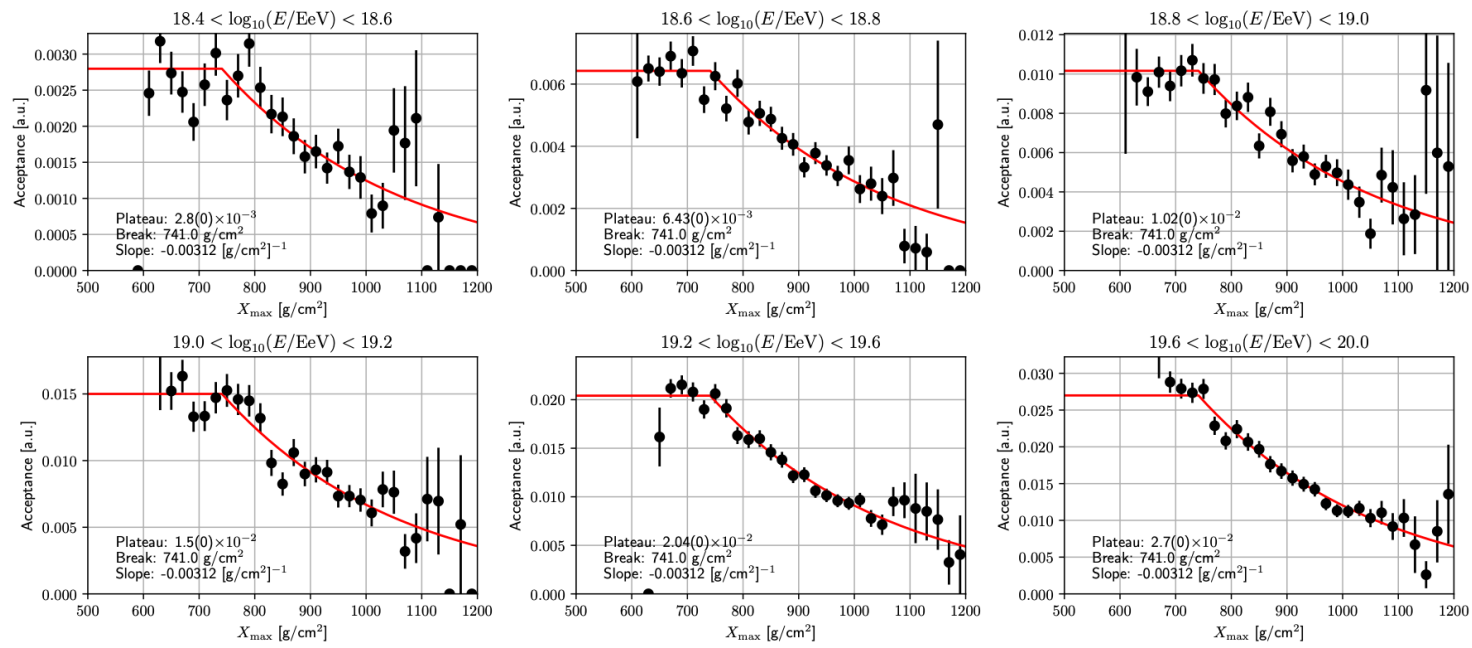

Figure 1: Stereo $X_{\max }$ acceptance calculated from QGSJetII-04 simulations of iron and proton primaries. The acceptance is fit to a model with a plateau at small $X_{\max }$, a break point in $X_{\max }$, and an exponential decrease in acceptance with $X_{\max }$ above the break point. The fits were performed using a binned loglikelihood minimization. The break point and exponential slope were required to be the same for all energy bins.

301 steps for $\log _{10} R_{\max }$ from $0-3$. The deviance contours corresponding to $10-\sigma$ are shown in Figure 2. The best fit point is at $(2.06,2.26)$ with a total deviance $D=126.0$ for $65=16+55-6$ degrees-of-freedom. There is also a local minimum at the point $(0.78,1.20)$ with $D=200.5$.

The details of the best fit point $\left(\gamma=2.06, R_{\max }=182 \mathrm{EV}\right.$ ) are shown in Figures 3 and 4 . The source fractions are: $0.0 \%, 99.2 \%, 0.0 \%, 0.0 \%$, and $0.8 \%$ for $\mathrm{H}, \mathrm{He}, \mathrm{N}, \mathrm{Si}$, and $\mathrm{Fe}$, respectively. The fit is dominated by the very large flux in TA above $10^{1.7} \mathrm{EeV}$ which is above the model's prediction for a GZK-effect.

The details of the fit for the secondary minimum $\left(\gamma=0.78, R_{\max }=15.8 \mathrm{EV}\right)$ are shown in Figures 5 and 6. The source fractions are: $61.0 \%, 37.4 \%, 1.5 \%, 0.1 \%$, and $0.003 \%$ for $\mathrm{H}, \mathrm{He}, \mathrm{N}$, $\mathrm{Si}$, and $\mathrm{Fe}$, respectively.

\section{Neutrino Flux Prediction}

Using CRPropa one can also simulate the production of neutrinos from the propagation of cosmic rays. We performed this calculation while generating the shell model of the cosmic rays for our model. We can then predict the cosmogenic neutrinos produced during the propagation of the cosmic rays used to fit the Telescope Array data. We produced cosmic rays from source distances beyond what is relevant for fitting the cosmic ray spectrum, but which will contribute to the neutrino flux. For these distances, $1<z<4$, we used the van Vliet et al.[8] prescription for the evolution of the sources, which is $(1+z)^{m}$ for $z<1.5$, and constant for $z>1.5$. The predicted flux is shown in Figure 7, and includes the sum of muon and electron neutrino and anti-neutrinos.

The higher cutoff energy of the TA cosmic ray spectrum thus open the possibility that there will be a considerable flux of cosmogenic neutrinos, a flux which is in the range of planned neutrino detectors such as ARIANNA-200. 


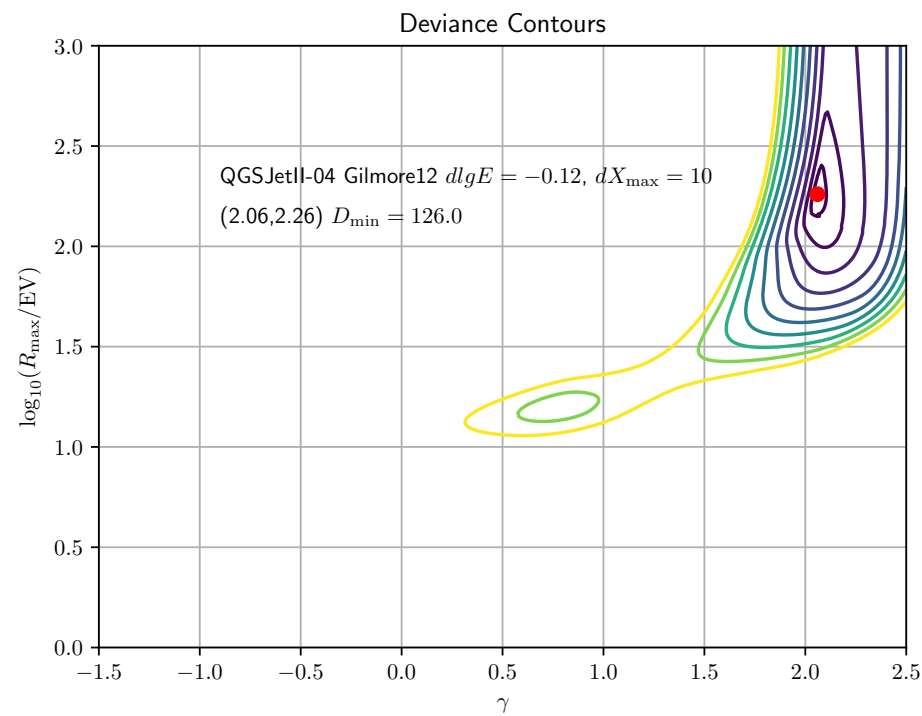

Figure 2: A plot of the deviance contours corresponding to 10- $\sigma$ for Telescope Array data using QGSJetII-04 as the high-energy interaction model and the Gilmore-12 IRB model, $\Delta \log _{10} E=-0.12$ and $\Delta X_{\max }=+10$ $\mathrm{g} / \mathrm{cm}^{2}(0.67 \sigma)$. The best fit point is $\gamma=2.06, \log _{10}\left(R_{\max } / \mathrm{EV}\right)=2.26\left(R_{\max }=182 \mathrm{EV}\right)$. This point had source fractions: $0.0 \%, 99.2 \%, 0.0 \%, 0.0 \%$, and $0.8 \%$ for $\mathrm{H}, \mathrm{He}, \mathrm{N}, \mathrm{Si}$, and $\mathrm{Fe}$, respectively. The local minimum at $\gamma=0.78, \log _{10}\left(R_{\max } / \mathrm{EV}\right)=1.20\left(R_{\max }=15.8 \mathrm{EV}\right)$ has source fractions: $97.1 \%, 0.0 \%, 0.0 \%$, $0.0 \%$, and $2.9 \%$ for $\mathrm{H}, \mathrm{He}, \mathrm{N}, \mathrm{Si}$, and $\mathrm{Fe}$, respectively.
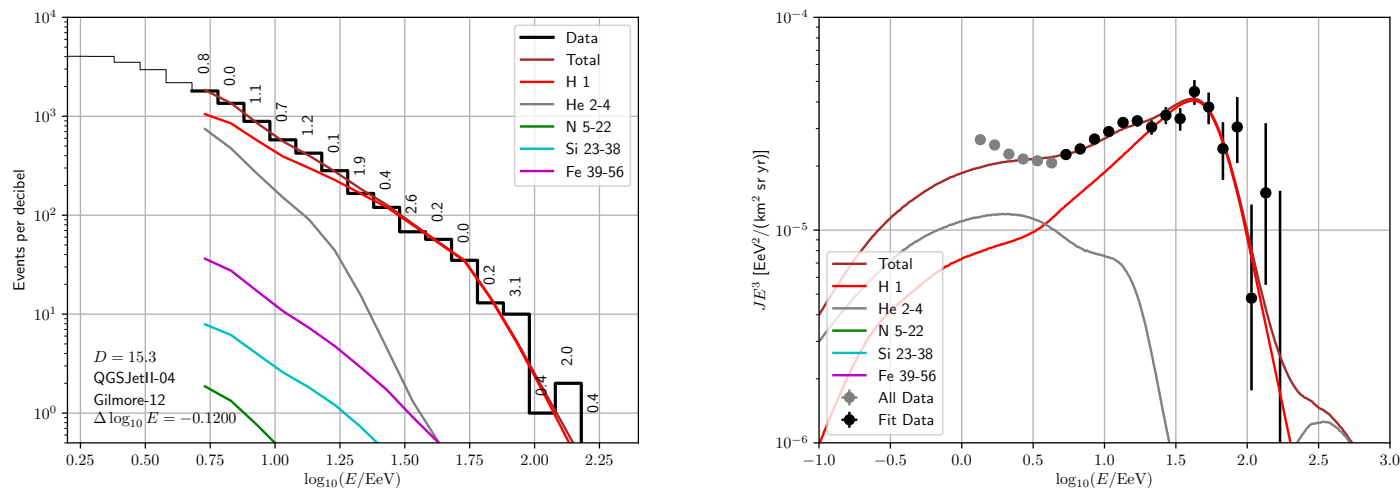

Figure 3: Left: The observed event energy distribution and the model predictions for observed species the best fit point. The fit gives a predominantly $\mathrm{H}$ at observation, despite no $\mathrm{H}$ at the source. There are 16 bins contributing to the degrees-of-freedom in the fit. Right: The observed and predicted energy flux spectra (multiplied by $E^{3}$ ) for the best fit. 

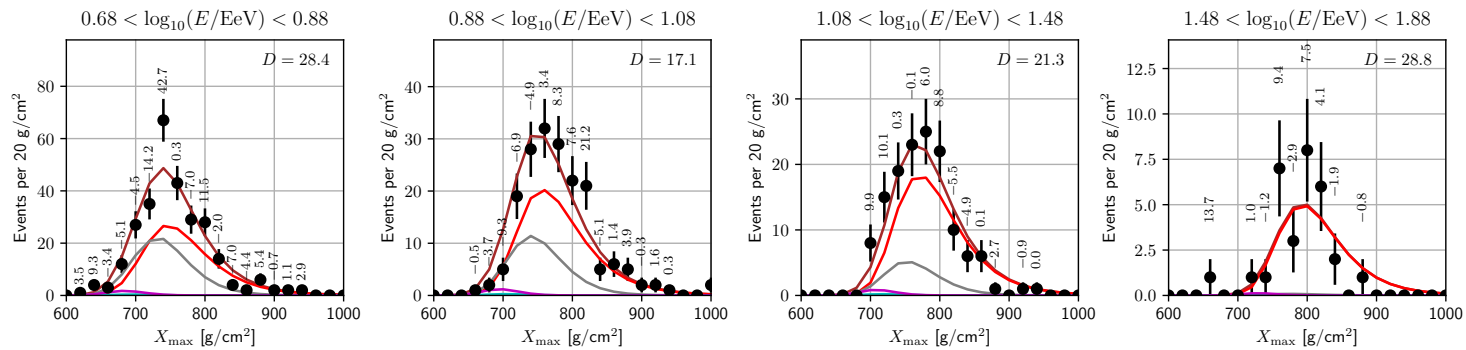

Figure 4: The observed $X_{\max }$ distributions in bins of energy along with the predicted $X_{\max }$ distributions from the model the best fit. The colors of the observed elemental group predictions is the same as in Figure 3. There are a total of 55 non-empty data bins contributing to the degrees-of-freedom
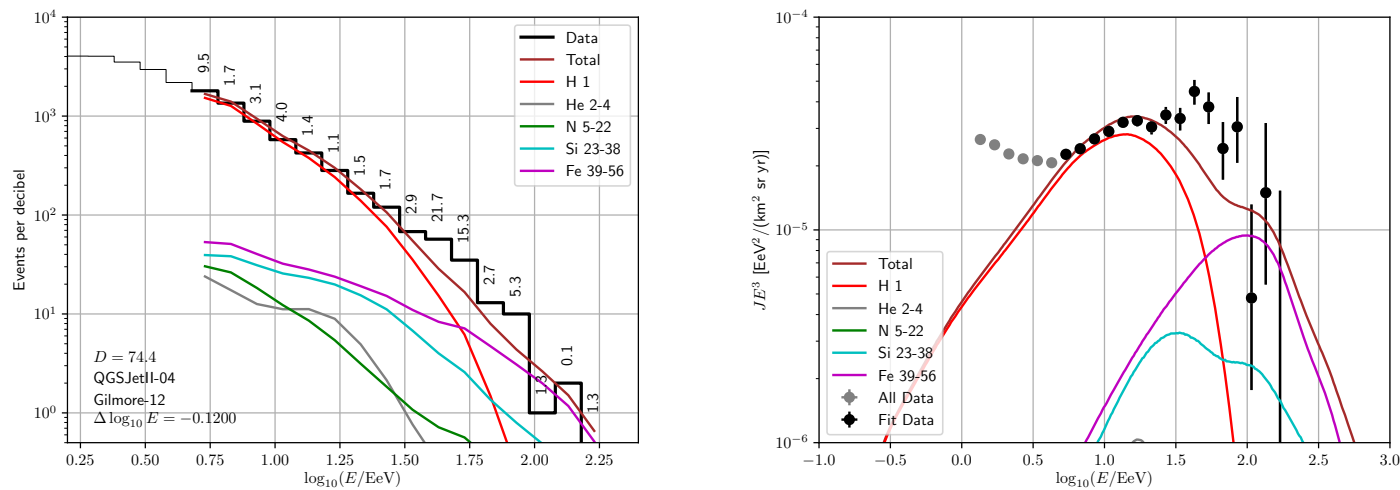

Figure 5: Left: The observed event energy distribution and the model predictions for observed species in the secondary minimum. Right: The observed and predicted energy flux spectra (multiplied by $E^{3}$ ) for the secondary minimum.
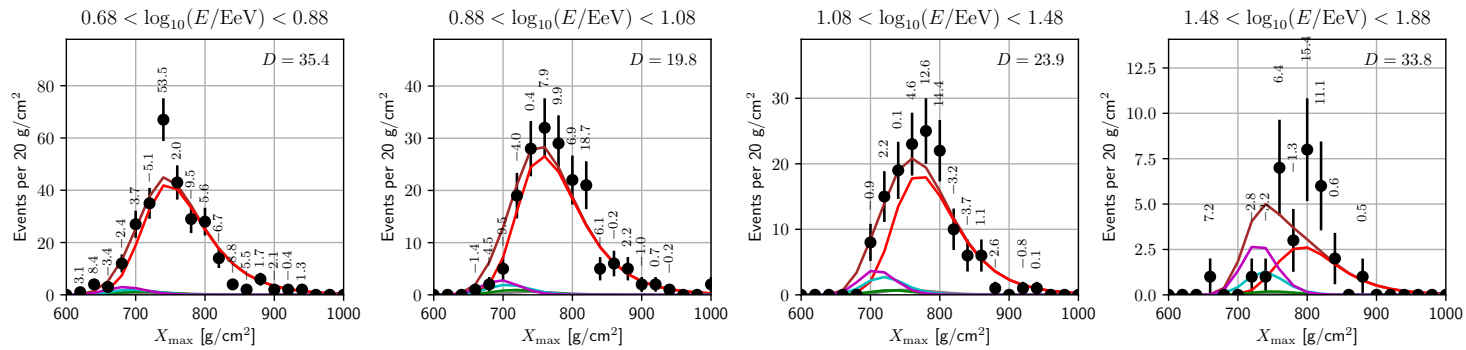

Figure 6: The observed $X_{\max }$ distributions in bins of energy along with the predicted $X_{\max }$ distributions from the model in the secondary minimum. The colors of the observed elemental group predictions is the same as in Figure 3. 


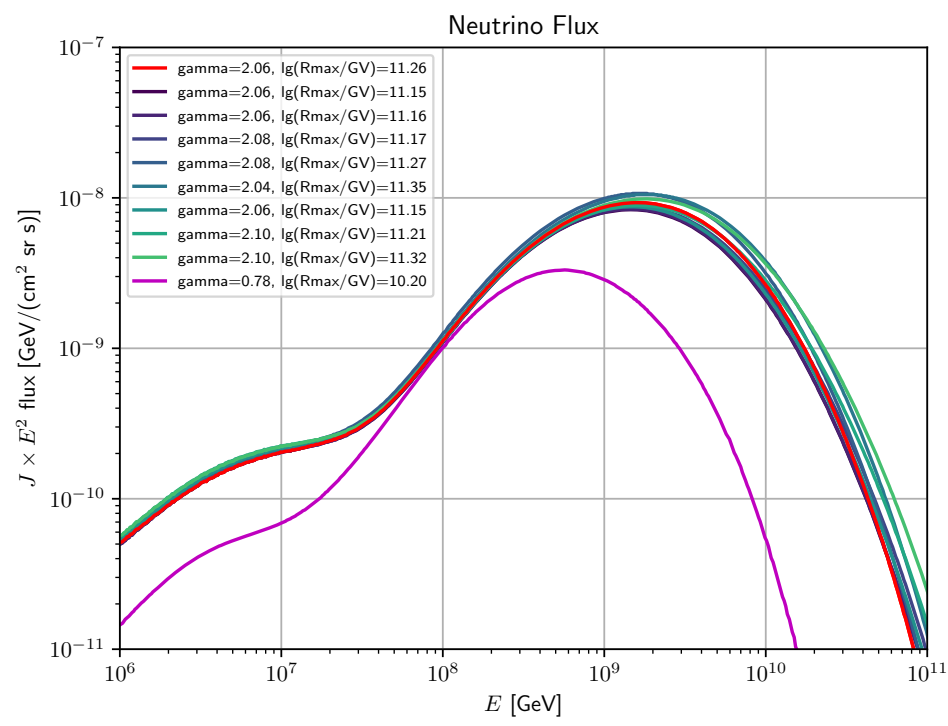

Figure 7: The predicted cosmogenic neutrino flux predicted from the source model of cosmic rays fit in this paper. Note that units are $\mathrm{GeV}$ and $\mathrm{GV}$ to align with neutrino astronomy conventions. The prediction from our best fit model is shown in red. We sampled fits from the scan around the 1- $\sigma$ contour to get generate an uncertainty band for the predicted flux. While the source is mostly helium, the high rigidity cutoff implies that many of the spalated protons and neutrons are still above the GZK-threshold energy and can thus contribute to the cosmogenic neutrino flux. The prediction of the neutrino flux from the secondary minimum is also shown, in magenta.

\section{Discussion}

From the fit results shown of the Telescope Array spectrum and stereo composition data, a number of different interpretations of source compositions could be drawn. Not all of these source compositions are astrophysically plausible, e.g., predominantly $\mathrm{He}$ with no $\mathrm{H}$; or predominantly $\mathrm{H}$ with no He nor $\mathrm{N}$ but a little Fe. The best fit for the TA data overall shows that one might expect predominantly $\mathrm{H}$ at observation although no $\mathrm{H}$ was accelerated at the source. This is quite different from the interpretation gleaned from the combined fit to the Auger data, which showed a moderately soft spectrum at the source contributing to a progressively heavier set of dominant species at observation. Since the Auger and TA spectrum data are consistent in this range except for the very highest energies, this difference in interpretation is very dependent on shape of the spectrum in the very high energy region.

\section{Acknowledgments}

The Telescope Array experiment is supported by the Japan Society for the Promotion of Science(JSPS) through Grants-in-Aid for Priority Area 431, for Specially Promoted Research JP21000002, for Scientific Research (S) JP19104006, for Specially Promoted Research JP15H05693, for Scientific Research (S) JP15H05741 and JP19H05607, for Science Research (A) JP18H03705, for Young Scientists (A) JPH26707011, and for Fostering Joint International Research (B) JP19KK0074, by the joint research program 
of the Institute for Cosmic Ray Research (ICRR), The University of Tokyo; by the Pioneering Program of RIKEN for the Evolution of Matter in the Universe (r-EMU); by the U.S. National Science Foundation awards PHY-1404495, PHY-1404502, PHY-1607727, PHY-1712517, PHY-1806797 and PHY-2012934; by the National Research Foundation of Korea (2017K1A4A3015188, 2020R1A2C1008230, \& 2020R1A2C2102800) ; by the Ministry of Science and Higher Education of the Russian Federation under the contract 075-15-2020778, RFBR grant 20-02-00625a (INR), IISN project No. 4.4501.18, and Belgian Science Policy under IUAP VII/37 (ULB). This work was partially supported by the grants ofThe joint research program of the Institute for Space-Earth Environmental Research, Nagoya University and Inter-University Research Program of the Institute for Cosmic Ray Research of University of Tokyo. The foundations of Dr. Ezekiel R. and Edna Wattis Dumke, Willard L. Eccles, and George S. and Dolores Doré Eccles all helped with generous donations. The State of Utah supported the project through its Economic Development Board, and the University of Utah through the Office of the Vice President for Research. The experimental site became available through the cooperation of the Utah School and Institutional Trust Lands Administration (SITLA), U.S. Bureau of Land Management (BLM), and the U.S. Air Force. We appreciate the assistance of the State of Utah and Fillmore offices of the BLM in crafting the Plan of Development for the site. Patrick A. Shea assisted the collaboration with valuable advice and supported the collaborationâ€ $€^{\mathrm{TM}_{\mathrm{S}}}$ efforts. The people and the officials of Millard County, Utah have been a source of steadfast and warm support for our work which we greatly appreciate. We are indebted to the Millard County Road Department for their efforts to maintain and clear the roads which get us to our sites. We gratefully acknowledge the contribution from the technical staffs of our home institutions. An allocation of computer time from the Center for High Performance Computing at the University of Utah is gratefully acknowledged.

\section{References}

[1] The Pierre Auger collaboration, Combined fit of spectrum and composition data as measured by the Pierre Auger Observatory, JCAP(2017)038. [arXiv:1612.07155]

[2] Y. Tsunesada et al., Energy Spectrum of Ultra-High-Energy Cosmic Rays Measured by The Telescope Array, PoS(ICRC2017)535.

[3] T. Stroman, D. Bergman, Telescope Array measurement of UHECR composition from stereoscopic fluorescence detection, PoS(ICRC2017)538.

[4] R.A. Batista et al., CRPropa 3 - a Public Astrophysical Simulation Frameworkfor Propagating Extraterrestrial Ultra-High Energy Particles, JCAP05(2016)038. [arXiv:1603.07142]

[5] R.C. Gilmore et al., Semi-analytic modeling of the EBL and consequences for extragalactic gamma-ray spectra, Mon. Not. Roy. Astron. Soc. 422 (2012) 3189. [arXiv:1104.0671]

[6] Planck Collaboration, Planck 2015 results. XIII. Cosmological parameters, A\&A 594, A13 (2016). [arXiv:1502.01589]

[7] M. De Dominico et al., Reinterpreting the development of extensive air showers initiated by nuclei and photons, JCAP07(2013)050. [arXiv:1305.2331]

[8] van Vliet et al., Determining the fraction of cosmic-ray protons at ultrahigh energies with cosmogenic neutrinos, Phys. Rev. D 100 (2019) 021302. [arXiv:1901.01899] 


\section{Full Authors List: Telescope Array Collaboration}

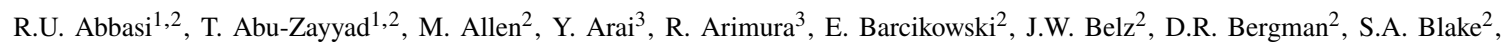
I. Buckland ${ }^{2}$, R. $\mathrm{Cady}^{2}$, B.G. Cheon ${ }^{4}$, J. Chiba ${ }^{5}$, M. Chikawa ${ }^{6}$, T. Fujii ${ }^{7}$, K. Fujisue ${ }^{6}$, K. Fujita ${ }^{3}$, R. Fujiwara ${ }^{3}$, M. Fukushima ${ }^{6}$, R. Fukushima ${ }^{3}$, G. Furlich ${ }^{2}$, R. Gonzalez ${ }^{2}$, W. Hanlon ${ }^{2}$, M. Hayashi ${ }^{8}$, N. Hayashida ${ }^{9}$, K. Hibino ${ }^{9}$, R. Higuchi ${ }^{6}$, K. Honda ${ }^{10}$, D. Ikeda ${ }^{9}$, T. Inadomi ${ }^{11}$, N. Inoue ${ }^{12}$, T. Ishii ${ }^{10}$, H. Ito ${ }^{13}$, D. Ivanov ${ }^{2}$, H. Iwakura ${ }^{11}$, A. Iwasaki ${ }^{3}$, H.M. Jeong ${ }^{14}$, S. Jeong ${ }^{14}$, C.C.H. Jui ${ }^{2}$, K. Kadota ${ }^{15}$, F. Kakimoto ${ }^{9}$, O. Kalashev ${ }^{16}$, K. Kasahara ${ }^{17}$, S. Kasami ${ }^{18}$, H. Kawai ${ }^{19}$, S. Kawakami ${ }^{3}$, S. Kawana ${ }^{12}$, K. Kawata ${ }^{6}$, I. Kharuk ${ }^{16}$, E. Kido ${ }^{13}$, H.B. Kim ${ }^{4}$, J.H. Kim ${ }^{2}$, J.H. Kim ${ }^{2}$, M.H. Kim ${ }^{14}$, S.W. Kim ${ }^{14}$, Y. Kimura ${ }^{3}$, S. Kishigami ${ }^{3}$, Y. Kubota ${ }^{11}$, S. Kurisu ${ }^{11}$, V. Kuzmin ${ }^{16}$, M. Kuznetsov ${ }^{16,20}$, Y.J. Kwon ${ }^{21}$, K.H. Lee ${ }^{14}$, B. Lubsandorzhiev ${ }^{16}$, J.P. Lundquist ${ }^{2,22}$, K. Machida ${ }^{10}$, H. Matsumiya ${ }^{3}$, T. Matsuyama ${ }^{3}$, J.N. Matthews ${ }^{2}$, R. Mayta ${ }^{3}$, M. Minamino ${ }^{3}$, K. Mukai ${ }^{10}$, I. Myers ${ }^{2}$, S. Nagataki ${ }^{13}$, K. Nakai ${ }^{3}$, R. Nakamura ${ }^{11}$, T. Nakamura ${ }^{23}$, T. Nakamura ${ }^{11}$, Y. Nakamura ${ }^{11}$, A. Nakazawa ${ }^{11}$, E. Nishio ${ }^{18}$, T. Nonaka ${ }^{6}$, H. Oda ${ }^{3}$, S. Ogio $^{3,24}$, M. Ohnishi ${ }^{6}$, H. Ohoka ${ }^{6}$, Y. Oku ${ }^{18}$, T. Okuda ${ }^{25}$, Y. Omura ${ }^{3}$, M. Ono ${ }^{13}$, R. Onogi ${ }^{3}$, A. Oshima ${ }^{3}$, S. Ozawa ${ }^{26}$, I.H. Park ${ }^{14}$, M. Potts ${ }^{2}$, M.S. Pshirkov ${ }^{16,27}$, J. Remington ${ }^{2}$, D.C. Rodriguez ${ }^{2}$, G.I. Rubtsov ${ }^{16}$, D. Ryu ${ }^{28}$, H. Sagawa ${ }^{6}$, R. Sahara ${ }^{3}$, Y. Saito ${ }^{11}$, N. Sakaki ${ }^{6}$, T. Sako ${ }^{6}$, N. Sakurai ${ }^{3}$, K. Sano ${ }^{11}$, K. Sato ${ }^{3}$, T. Seki ${ }^{11}$, K. Sekino ${ }^{6}$, P.D. Shah ${ }^{2}$, Y. Shibasaki ${ }^{11}$, F. Shibata ${ }^{10}$, N. Shibata ${ }^{18}$, T. Shibata ${ }^{6}$, H. Shimodaira ${ }^{6}$, B.K. Shin ${ }^{28}$, H.S. Shin ${ }^{6}$, D. Shinto ${ }^{18}$, J.D. Smith ${ }^{2}$, P. Sokolsky ${ }^{2}$, N. Sone ${ }^{11}$, B.T. Stokes ${ }^{2}$, T.A. Stroman ${ }^{2}$, Y. Takagi ${ }^{3}$,

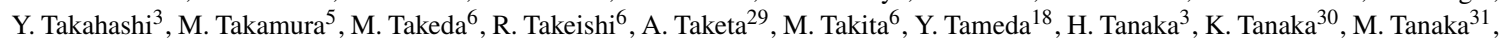
Y. Tanoue ${ }^{3}$, S.B. Thomas ${ }^{2}$, G.B. Thomson ${ }^{2}$, P. Tinyakov ${ }^{16,20}$, I. Tkachev ${ }^{16}$, H. Tokuno ${ }^{32}$, T. Tomida ${ }^{11}$, S. Troitsky ${ }^{16}$, R. Tsuda ${ }^{3}$, Y. Tsunesada ${ }^{3,24}$, Y. Uchihori ${ }^{33}$, S. Udo ${ }^{9}$, T. Uehama ${ }^{11}$, F. Urban ${ }^{34}$, T. Wong ${ }^{2}$, K. Yada $^{6}$, M. Yamamoto ${ }^{11}$, K. Yamazaki ${ }^{9}$, J. Yang ${ }^{35}$, K. Yashiro ${ }^{5}$, F. Yoshida ${ }^{18}$, Y. Yoshioka ${ }^{11}$, Y. Zhezher ${ }^{6,16}$, and Z. Zundel ${ }^{2}$

${ }^{1}$ Department of Physics, Loyola University Chicago, Chicago, Illinois, USA

${ }^{2}$ High Energy Astrophysics Institute and Department of Physics and Astronomy, University of Utah, Salt Lake City, Utah, USA

${ }^{3}$ Graduate School of Science, Osaka City University, Osaka, Osaka, Japan

${ }^{4}$ Department of Physics and The Research Institute of Natural Science, Hanyang University, Seongdong-gu, Seoul, Korea

${ }^{5}$ Department of Physics, Tokyo University of Science, Noda, Chiba, Japan

${ }^{6}$ Institute for Cosmic Ray Research, University of Tokyo, Kashiwa, Chiba, Japan

7 The Hakubi Center for Advanced Research and Graduate School of Science, Kyoto University, KitashirakawaOiwakecho, Sakyo-ku, Kyoto, Japan

${ }^{8}$ Information Engineering Graduate School of Science and Technology, Shinshu University, Nagano, Nagano, Japan

${ }^{9}$ Faculty of Engineering, Kanagawa University, Yokohama, Kanagawa, Japan

${ }^{10}$ Interdisciplinary Graduate School of Medicine and Engineering, University of Yamanashi, Kofu, Yamanashi, Japan

${ }^{11}$ Academic Assembly School of Science and Technology Institute of Engineering, Shinshu University, Nagano, Nagano, Japan

${ }^{12}$ The Graduate School of Science and Engineering, Saitama University, Saitama, Saitama, Japan

13 Astrophysical Big Bang Laboratory, RIKEN, Wako, Saitama, Japan

${ }^{14}$ Department of Physics, SungKyunKwan University, Jang-an-gu, Suwon, Korea

${ }^{15}$ Department of Physics, Tokyo City University, Setagaya-ku, Tokyo, Japan

${ }^{16}$ Institute for Nuclear Research of the Russian Academy of Sciences, Moscow, Russia

${ }^{17}$ Faculty of Systems Engineering and Science, Shibaura Institute of Technology, Minato-ku, Tokyo, Japan

${ }^{18}$ Department of Engineering Science, Faculty of Engineering, Osaka Electro-Communication University, Neyagawashi, Osaka, Japan

${ }^{19}$ Department of Physics, Chiba University, Chiba, Chiba, Japan

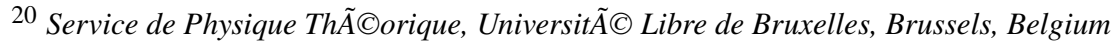

${ }^{21}$ Department of Physics, Yonsei University, Seodaemun-gu, Seoul, Korea

22 Center for Astrophysics and Cosmology, University of Nova Gorica, Nova Gorica, Slovenia

${ }^{23}$ Faculty of Science, Kochi University, Kochi, Kochi, Japan

${ }^{24}$ Nambu Yoichiro Institute of Theoretical and Experimental Physics, Osaka City University, Osaka, Osaka, Japan

${ }^{25}$ Department of Physical Sciences, Ritsumeikan University, Kusatsu, Shiga, Japan

${ }^{26}$ Quantum ICT Advanced Development Center, National Institute for Information and Communications Technology, Koganei, Tokyo, Japan

27 Sternberg Astronomical Institute, Moscow M.V. Lomonosov State University, Moscow, Russia

${ }^{28}$ Department of Physics, School of Natural Sciences, Ulsan National Institute of Science and Technology, UNIST-gil, 
Ulsan, Korea

${ }^{29}$ Earthquake Research Institute, University of Tokyo, Bunkyo-ku, Tokyo, Japan

${ }^{30}$ Graduate School of Information Sciences, Hiroshima City University, Hiroshima, Hiroshima, Japan

${ }^{31}$ Institute of Particle and Nuclear Studies, KEK, Tsukuba, Ibaraki, Japan

${ }^{32}$ Graduate School of Science and Engineering, Tokyo Institute of Technology, Meguro, Tokyo, Japan

33 Department of Research Planning and Promotion, Quantum Medical Science Directorate, National Institutes for Quantum and Radiological Science and Technology, Chiba, Chiba, Japan

${ }^{34}$ CEICO, Institute of Physics, Czech Academy of Sciences, Prague, Czech Republic

${ }^{35}$ Department of Physics and Institute for the Early Universe, Ewha Womans University, Seodaaemun-gu, Seoul, Korea

${ }^{*}$ Deceased 